\title{
Erratum
}

\section{Erratum: Fairhall, “Cross Recruitment of Domain-Selective Cortical Representations Enables Flexible Semantic Knowledge"}

In the article, "Cross Recruitment of Domain-Selective Cortical Representations Enables Flexible Semantic Knowledge," by Scott L. Fairhall, which appeared on pages 3096-3103 of the April 8, 2020 issue, errors were published.

The description of the initial stimulus selection includes incorrect details. In the fourth paragraph of the Materials and Methods section, the first sentence has been corrected to "The 32 stimuli in each category employed in the experiment were selected from an initial pool of 69 well-known food dishes, 115 famous people, and 92 Italian cities. The final ratings for each included stimulus were based on the responses of 20 participants for 83 of the included items and 12 responses for the remaining 13 items." The sentence previously incorrectly stated the details "50 well-known food dishes," "50 Italian cities," and "30 local people" participating.

In the first paragraph of the Materials and Methods section, the third sentence has been corrected to "Four participants were excluded because head motion exceeded $3.0 \mathrm{~mm}$." The sentence previously incorrectly stated that the within-run head-motion threshold used to exclude participants was $2.5 \mathrm{~mm}$.

One participant was excluded before preprocessing; because of a record-keeping failure, the reason for this exclusion is uncertain. However, this participant does not affect the significance or nature of any of the results reported in the paper.

The online version has been corrected.

DOI: 10.1523/JNEUROSCI.0609-21.2021 